\title{
Impact of sleep disordered breathing on behaviour among elementary school-aged children: a cross-sectional analysis of a large community-based sample
}

\author{
Dale L. Smith ${ }^{1,2}$, David Gozal ${ }^{3}$, Scott J. Hunter ${ }^{3,4}$, Mona F. Philby ${ }^{3}$, \\ Jaeson Kaylegian ${ }^{4}$ and Leila Kheirandish-Gozal ${ }^{3}$
}

Affiliations: ${ }^{1}$ Dept of Public Health Sciences, Pritzker School of Medicine, Biological Sciences Division, The University of Chicago, Chicago, IL, USA. ${ }^{2}$ Behavioral Sciences, College of Arts and Sciences, Olivet Nazarene University, Bourbonnais, IL, USA. ${ }^{3}$ Dept of Pediatrics, Pritzker School of Medicine, Biological Sciences Division, The University of Chicago, Chicago, IL, USA. "Dept of Psychiatry and Behavioral Neuroscience, Pritzker School of Medicine, Biological Sciences Division, The University of Chicago, Chicago, IL, USA.

Correspondence: Leila Kheirandish-Gozal, Section of Pediatric Sleep Medicine, Dept of Pediatrics, Pritzker School of Medicine, Biological Sciences Division, The University of Chicago, $5841 \mathrm{~S}$. Maryland Avenue, Office C-113/MC2117, Chicago, IL 60637-1470, USA. E-mail: Igozallapeds.bsd.uchicago.edu

ABSTRACT Sleep disordered breathing (SDB) in children has been associated with inattention, impulsivity and hyperactivity, but the associations between SDB severity and the type and severity of behavioural disruption are unclear.

1022 children aged 5-7 years old prospectively underwent sleep studies and behavioural assessments through completion of standardised instruments. Participants were subdivided into four categorical groups based on the apnoea-hypopnoea index (AHI; measured per hour of total sleep time (hTST)), i.e. Group 1: nonsnoring and AHI $<1 \mathrm{hTST}^{-1}$; Group 2: habitual snoring and AHI $<1 \mathrm{hTST}^{-1}$; Group 3: habitual snoring and AHI 1-5 hTST ${ }^{-1}$; and Group 4: habitual snoring and AHI $>5 \mathrm{hTST}^{-1}$, followed by comparisons of behavioural functioning across the groups.

All 10 behavioural variables differed significantly between Group 1 and all other groups. Post hoc comparisons indicated that Group 2 was the most impaired for most behavioural measures. Furthermore, differences between Group 2 and more severe sleep pathology conditions were rarely significant.

This large community-based paediatric cohort confirms earlier findings highlighting a significant impact of SDB on behavioural regulation, with the greatest impact being already apparent among habitually snoring children. Thus, a likely low asymptote exists regarding SDB behavioural impact, such that further increases in severity do not measurably increase parent-rated difficulties with behavioural regulation relative to controls. Our findings do support the need for considering early intervention, particularly among those children manifesting a behavioural impact of SDB.

@ERSpublications

Snoring in school-aged children, even without sleep/respiratory disturbances, is linked to behavioural disturbances http://ow.ly/G4xJ302JlLF 


\section{Introduction}

Sleep disordered breathing (SDB) is characterised by recurrent episodes of narrowing or collapse of the upper airway that may lead to intermittent hypoxaemia and hypercapnia, ultimately resulting in sleep fragmentation and nonrestorative sleep [1]. The prevalence of obstructive sleep apnoea (OSA) in children, the more severe form of SDB, ranges between 1 and 5\%, but habitual snoring is much more frequent and may affect up to $27 \%$ of young and school-aged children $[2,3]$ with a median of $11-12 \%$ being reported worldwide [4-8]. In children, one of the major consequences consistently reported in the presence of SDB consists of the emergence of behavioural disturbances, such as restlessness, aggressive behaviour, excessive daytime sleepiness and poor educational outcomes [9-17]. However, results have been variable across studies, specifically with regard to the patterns of behavioural impact seen at differing levels of SDB. Importantly though, compelling evidence to support a causative association between SDB and hyperactivity and inattentive behaviours in children has emerged in the last two decades [18-23], highlighting a significant relationship between SDB and the pattern of symptoms often leading to a diagnosis of attention-deficit/ hyperactivity disorder (ADHD) on the part of paediatricians and child psychiatrists or psychologists. In a recent and the only randomised clinical trial to date, children who were randomised to receive early adenotonsillectomy for SDB demonstrated improvement in behavioural functioning at 7 months follow-up relative to controls [24]. Additionally, daytime sleepiness, hyperactivity and aggressive behaviours have all been documented in children who habitually snore, even in the absence of SDB [25-27].

As would be predicted from a mechanistic association, objectively measured sleep and respiratory disturbances have been found to be relatively frequent among children with ADHD-like behaviours [28-30]. Although the exact mechanisms by which SDB elicits hyperactivity and inattention remain unknown, it is possible that the sleep fragmentation and episodic hypoxia that characterise SDB lead to alterations within the neurochemical substrate of the pre-frontal cortex and its connections with regulatory subcortical structures, with resultant executive and attentional dysfunction [31]. Indeed, snoring children were found to perform poorly on tests aimed at assessing the ability to develop and sustain an organised, future-oriented and flexible approach to problem solving, and alterations in sustained and divided attention have been reported consistently [32,33]. Furthermore, the manifestations of excessive daytime sleepiness differ in children compared with adults, such that both inattention and hyperactivity constitute significant behavioural correlates of sleepiness in younger patients [34-36].

We should, however, point out that the majority of the published studies have assessed relatively restricted paediatric cohorts, and have shown significant variability across measures and approaches taken with such studies. This has fostered remaining questions regarding both the absolute frequency of behavioural difficulties in children with SDB and the relationship of SDB severity with the magnitude of the symptoms presented in affected children.

Based on the standing literature regarding the potential neurodevelopmental mechanisms underlying the regulatory disruptions observed, we hypothesised that behavioural disturbances would be more prominent in children with any form of SDB, i.e. independent of the spectrum of SDB severity. Through examination of a large cohort of community-recruited children, spanning a wide range of the SDB severity spectrum, we anticipated that challenges in attention and regulation would already be evident at the lower levels of SDB severity, and that greater SDB severity would not yield any incremental behavioural impact.

\section{Methods}

\section{Participants}

The prospective paediatric cohort included children aged 5-7 years old residing in Louisville, KY, USA (recruited from October 2006 to November 2008) and children residing in Chicago, IL, USA (recruited from May 2009 to October 2014). The study was approved by both the University of Louisville (protocol 474.99) and the University of Chicago (protocol 09-115-B) Human Research Ethics Committees. Informed consent was obtained from the parents or legal caretakers of the children and age-appropriate assent was also obtained from the children themselves.

\section{Questionnaires}

Sleep status

Parents of all participating children were invited to complete a detailed and validated questionnaire about their child's sleeping habits as described previously [30, 37, 38]. The information gathered through the questionnaire included sex, age, ethnic background, questions on whether the child had difficulty breathing during sleep, mouth breathing, witnessed apnoea and daytime sleepiness. Snoring and the severity of the snoring were also included. For the question on snoring, the responses were graded as "never," "rarely" (once per week), "occasionally" (twice per week), "frequently" (three times per week) and "almost alwaysalways" (four or more times per week). Returned questionnaires were scanned into a computerised database 
and were subdivided according to their snoring patterns into nonsnoring children (responses of never or rarely on snore and not applicable on loudness of snore in questionnaire) or habitually snoring children (responses of frequently or almost always-always (three or more nights per week) on snoring frequency and medium loud to loud on loudness of snoring). Based on the responses, children were able to be identified and effectively grouped into those with and without multiple indices of SDB severity.

\section{Behaviour}

Child Behavior Checklist - Revised

The Child Behavior Checklist - Revised (CBCL) is the most commonly used parent report scale for measuring problem behaviours and mood challenges in children. For this study, the original version of the CBCL - Parent Report for youth aged 5-17 years old was used [39]. This version includes eight empirically derived syndrome subscales (Withdrawn, Somatic Complaints, Anxious/Depressed, Social Problems, Thought Problems, Attention-Hyperactivity Problems, Delinquent Behaviour and Aggressive Behaviour), two partial summary scales (Internalising Problems (a combination of the Withdrawn, Somatic Complaints and Anxious/Depressed subscales) and Externalising Problems (a combination of the Delinquent Behaviour and Aggressive Behaviour subscales)) and a Total Problems scale. Test-retest reliability and internal consistency are in the range very good to excellent [40]. The CBCL yields T-scores with a mean \pm SD of $50 \pm 10$ for the principle Internalising Problems, Externalising Problems and Total Problems indices that were examined with this study. For the individual scales, the lower end of the $\mathrm{T}$ distribution is truncated at 50 (corresponding to the 50th percentile).

\section{Conners' Parent Rating Scales - Revised}

The Conners' Parent Rating Scales - Revised (CPRS-R) [41] is also a parent report scale, examining the presence and severity of problem behaviours observed in children aged 3-17 years old. It was used in addition to the CBCL because it provides a more specific measure of attention problems in children, which is of particular relevance to this study given the findings that children with SDB frequently meet criteria for a diagnosis of an attentional disorder, more often than unaffected peers. Subscales of the CPRS include Oppositional, Cognitive Problems/Inattention, Hyperactivity, Anxious-Shy, Perfectionism, Social Problems and Psychosomatic ratings. Several summary measures are provided, including an ADHD Index, three Global Indices (Restless-Impulsive, Emotional Lability and Total), and three Diagnostic and Statistical Manual of Mental Disorders, Fourth Edition, Text Revision (DSM-IV-TR)-based symptom measures (Inattentive, Hyperactive-Impulsive and Total). Indices are standardised as T-scores, with a mean $\pm \mathrm{SD}$ of $50 \pm 10$. Test-retest reliability and internal consistency are adequate to excellent [42]. Additionally, a recent meta-analysis [43] supported the utility of both the CBCL and the CPRS for detection of ADHD symptoms.

\section{Overnight polysomnographic studies}

All children underwent standard nocturnal polysomnography (PSG) evaluation as described previously [44], with assessment of eight standard electroencephalography channels, bilateral electro-oculography, electromyography, two-lead electrocardiography, oronasal airflow measurement using a thermistor, nasal pressure transducer and end-tidal carbon dioxide, chest and abdominal movement by respiratory inductance plethysmography, and pulse oximetry including pulse waveform using a commercially available data acquisition system (Polysmith; Nihon Kohden America, Foothill Ranch, CA, USA), as described previously [44]. Each of the nocturnal PSG studies were scored as per the 2012 American Association of Sleep Medicine guidelines for the scoring of sleep and associated events by paediatric sleep experts (D. Gozal, M.F. Philby and L. KheirandishGozal) who were blind to the behavioural test results [45]. The proportion of time spent in each stage of sleep was calculated as a percentage of total sleep time (TST). A respiratory event was scored as an obstructive apnoea if it was associated with a $>90 \%$ decrease in signal amplitude for $>90 \%$ of the entire event compared with the baseline amplitude, the event lasted for at least two breaths and there was continued or increased respiratory effort throughout the period of the event. A mixed apnoea was scored if there was absent inspiratory effort in the initial part of the event, followed by resumption of inspiratory effort before the end of the event. A central apnoea was scored if respiratory effort was absent throughout the duration of the event, the event lasted for at least two missed breaths and was associated with an arousal/awakening or a $\geqslant 3 \%$ desaturation. A hypopnoea was scored if the event was associated with a $\geqslant 50 \%$ fall in amplitude of the nasal pressure transducer, lasted for at least two breaths and was associated with an arousal/awakening or $\geqslant 3 \%$ desaturation.

\section{Anthropometry}

During the nocturnal PSG test, height was measured with a stadiometer and recorded to the nearest $0.1 \mathrm{~cm}$. Weight was recorded to the nearest $0.1 \mathrm{~kg}$. Height and weight centiles were calculated using the Centre for Disease Control 2000 growth charts for the USA (www.cdc.gov/growthcharts). Body mass index (BMI) z-scores were calculated using The Children's Hospital of Philadelphia BMI and z-score calculation in children online software (stokes.chop.edu/web/zscore). 


\section{Data analysis}

Based on questionnaires and nocturnal PSG findings, children enrolled in the study were subdivided into four categorical groups based on recognised OSA severity levels [46] measured using the apnoeahypopnoea index (AHI; measured per hour of TST (hTST)), i.e. Group 1: nonsnoring and AHI $<1 \mathrm{hTST}^{-1}$; Group 2: habitual snoring and AHI $<1 \mathrm{hTST}^{-1}$; Group 3: habitual snoring and AHI $1-5 \mathrm{hTST}^{-1}$; and Group 4: habitual snoring and $\mathrm{AHI}>5 \mathrm{hTST}^{-1}$. Comparisons of behavioural functioning across the four snore/AHI groups were conducted using subtests of the CBCL and CPRS-R as described above. We selected 10 behavioural indices from the two questionnaires for comparison across the four groups based on the specific behavioural areas covered by these items; these included the primary indices from the CBCL (Internalising, Externalising, ADHD and Total scores) and the CPRS-R (Cognitive, Hyperactivity, Psychosomatic, ADHD Total, and Inattentive, Hyperactive-Impulsive and Total DSM-IV-TR criteria met). Standardised scores were used for all analyses. Homogeneity of variance across conditions was supported in all comparisons but two, but because of these exceptions, and departures from normality in some behavioural measures, Welch's ANOVA results are reported here. Multivariate procedures were not used due to the amount of missing data across measures, our inclusion of CPRS-R and CBCL scores derived from subscores that were also examined, departures from homogeneity of variance/covariance matrices and our interest in examining all behavioural measures. ANOVA results were determined to be significant at the $\mathrm{p}<0.05$ significance level, although we used Bonferroni's correction to control error rate across all omnibus ANOVAs due to the number of outcome measures. Following significant omnibus ANOVA results, we also used Bonferroni's correction for post hoc tests and adjusted p-values are reported for the results.

\section{Results}

From October 2006 to October 2014, 1022 children were recruited from public schools and completed the designated protocol. Specific subject characteristics are detailed in table 1. An initial comparison of mean BMI scores across these groups reached significance, as did proportion of Caucasian and African-American children in each group $(\mathrm{p}<0.001)$. Otherwise no significant differences were identified for potential confounders, although sleep variables differed as expected, and are presented here purely for illustrative purposes.

TABLE 1 General characteristics of the four groups of children based on the presence or absence of habitual snoring and their polysomnographically derived apnoea-hypopnoea indices (AHIs) (see main text for group definitions)

\begin{tabular}{|c|c|c|c|c|c|}
\hline & Group 1 & Group 2 & Group 3 & Group 4 & p-value \\
\hline Subjects & 87 & 436 & 359 & 140 & \\
\hline Age years & $6.88 \pm 0.67$ & $6.86 \pm 0.79$ & $6.92 \pm 0.99$ & $6.70 \pm 1.01$ & 0.176 \\
\hline Male & 55.56 & 55.25 & 54.17 & 56.74 & 0.953 \\
\hline \multicolumn{6}{|l|}{ Ethnicity } \\
\hline Caucasian & 75.9 & 64.2 & 54.7 & 29.9 & $<0.001^{\#}$ \\
\hline African-American & 13.8 & 28.8 & 32.8 & 58.2 & \\
\hline BMI z-score & $0.43 \pm 1.31$ & $0.66 \pm 1.26$ & $0.75 \pm 1.42$ & $1.26 \pm 1.53$ & $<0.001^{\#}$ \\
\hline \multicolumn{6}{|l|}{ Receiving } \\
\hline Montelukast & 8.0 & 14.1 & 13.9 & 17.8 & 0.402 \\
\hline Nasal corticosteroids & 6.9 & 8.7 & 8.9 & 7.9 & 0.642 \\
\hline TST min & $470.86 \pm 45.11$ & $471.71 \pm 44.21$ & $469.30 \pm 50.00$ & $460.08 \pm 55.27$ & 0.105 \\
\hline Sleep efficiency & $89.03 \pm 7.90$ & $89.15 \pm 7.67$ & $90.04 \pm 7.68$ & $89.68 \pm 8.33$ & 0.397 \\
\hline Stage 1 & $7.23 \pm 4.96$ & $6.88 \pm 4.89$ & $6.58 \pm 6.35$ & $7.83 \pm 6.46$ & 0.241 \\
\hline Stage 2 & $45.12 \pm 7.90$ & $45.96 \pm 7.92$ & $44.78 \pm 8.63$ & $44.39 \pm 7.69$ & 0.106 \\
\hline Stage SWS & $27.38 \pm 7.26$ & $26.32 \pm 9.04$ & $29.28 \pm 8.97$ & $26.34 \pm 8.58$ & 0.114 \\
\hline REM sleep & $24.50 \pm 14.78$ & $21.50 \pm 8.75$ & $20.68 \pm 8.48$ & $21.61 \pm 13.91$ & 0.096 \\
\hline Sleep latency min & $26.43 \pm 28.89$ & $23.99 \pm 24.55$ & $21.51 \pm 22.37$ & $18.19 \pm 21.66$ & 0.026 \\
\hline REM latency min & $145.55 \pm 64.53$ & $141.66 \pm 59.13$ & $151.59 \pm 68.55$ & $146.88 \pm 67.48$ & 0.192 \\
\hline Obstructive AHI hTST ${ }^{-1}$ & $0.39 \pm 0.29$ & $0.40 \pm 0.29$ & $2.11 \pm 1.03$ & $14.87 \pm 12.44$ & $<0.001^{\#}$ \\
\hline $\mathrm{SpO}_{2}$ nadir & $92.77 \pm 10.16$ & $92.48 \pm 7.49$ & $90.47 \pm 6.63$ & $82.50 \pm 10.95$ & $<0.001^{\#}$ \\
\hline$O D I \geqslant 3 \%$ & $0.01 \pm 0.01$ & $0.02 \pm 0.01$ & $1.96 \pm 0.77$ & $11.63 \pm 8.73$ & $<0.01^{\#}$ \\
\hline Total arousal index $\mathrm{hTST}^{-1}$ & $8.57 \pm 3.95$ & $9.12 \pm 5.76$ & $10.96 \pm 7.65$ & $17.56 \pm 11.27$ & $<0.001^{\#}$ \\
\hline Asthma & 13.79 & 20.69 & 18.85 & 24.42 & 0.323 \\
\hline
\end{tabular}

Data are presented as $n$, mean \pm SD or $\%$, unless otherwise stated. BMI: body mass index; TST: total sleep time; SWS: slow wave sleep; REM: rapid eye movement; $\mathrm{SpO}_{2}$ : arterial oxygen saturation measured by pulse oximetry; ODI: oxygen desaturation index. " omnibus comparison (Chi-squared for proportions, ANOVA for continuous variables) is significant after false discovery rate control for multiple comparisons [48]. 
All 10 behavioural variables differed significantly across snore/AHI levels (tables 2 and 3). The snoring/ AHI $<1$ hTST $^{-1}$ group (Group 2) suffered from the highest levels of behavioural pathology in all behavioural measures but one. This group was significantly more impaired than the control/nonsnoring group (Group 1) in all measures except CPRS-R Hyperactivity $(p=0.078)$. Group 2 was significantly more impaired than those with greater sleep pathology only for CBCL ADHD ( $p<0.001$ and $p=0.041$ for Group 3 and 4 comparisons, respectively), CPRS-R Cognitive ( $\mathrm{p}=0.048$ for Group 3 comparison) and CPRS-R Hyperactivity ( $\mathrm{p}=0.04$ and $\mathrm{p}=0.037$ for Group 3 and 4 comparison, respectively).

The tendency for Group 2 to demonstrate greater behavioural pathology was consistent across different ethnic groups, asthma status groups and sexes, although insufficient representation in Group 1 precluded further analysis of interaction effects or stratification by ethnicity.

Ethnicity (including only Caucasian and African-American children due to sample size limitations for other groups) was also included as a factor in separate analyses to determine the presence of interactions between ethnicity and AHI group for each behavioural measure. Interactions between AHI and ethnicity were significant for several behavioural measures, although trends were inconsistent and suffered from power limitations due to the small number of African-American children in the sample who did not snore $(n=13)$, and are not the focus of the current paper. Similar analyses using asthma as a second factor revealed interactions for several behavioural variables, although this was primarily due to greater behavioural problems in the asthmatic control group (Group 1), which could be spurious due to the very small sample of nonsnoring asthmatic children $(n=13)$. No such interactions were found for sex. Furthermore, analyses were conducted to examine the differences in behavioural functioning across AHI levels while controlling for age and BMI, which did not suggest any change in trends or significance patterns outlined above, and interactions between these variables and SDB were not significant.

When stratifying by BMI, we found similarity of trends for both normal BMI z-score $(<1.24)$ and high BMI z-score $(>1.64)$ children with regard to greater behavioural pathology for Group 2 than Group 1 children. It appears that for higher BMI z-score children, the pattern of reported behaviour problems remain fairly constant across all snoring groups, i.e. Groups 2-4. However, for children with low BMI z-scores, behaviour problems are more prevalent for Group 2 than for either Groups 3 or 4, although these differences were inconsistent, similar to the overall analysis above.

The trends and significance patterns from stratified analysis results did not differ notably from overall analyses reported here, so they were not included in the current paper. Additionally, BMI and AHI were also treated as continuous covariates in regression analyses during initial screening. However, neither BMI nor $\mathrm{BMI} \times \mathrm{AHI}$ interaction terms were typically significant as predictors of behavioural measures. As a result, these analyses were also excluded from the current paper.

Trends illustrating higher pathology for Group 2 appear fairly consistent across all significant behavioural measures. This generally suggests that across demographic and anthropomorphic conditions Group 2 was associated with a greater number of parent-reported behavioural problems than seen with nonsnoring children, although behavioural trends at higher levels of sleep pathology may be dependent on other factors.

TABLE 2 Behavioural functioning differences across snore/apnoea-hypopnoea index levels

\begin{tabular}{lccr} 
Test & $\mathbf{n}$ & Test statistic $\mathbf{F}$ & p-value \\
\hline CBCL Internalising & 989 & 5.27 & $0.001^{\#}$ \\
ADHD & 650 & 8.11 & $<0.001^{\#}$ \\
Total & 989 & 5.27 & $0.001^{\#}$ \\
CPRS-R Cognitive & 1021 & 4.80 & $0.003^{\#}$ \\
Hyperactivity & 1020 & 4.76 & $0.003^{\#}$ \\
Psychosomatic & 1022 & 5.92 & $0.001^{\#}$ \\
ADHD & 1022 & 5.24 & $0.001^{\#}$ \\
DSM Inattentive & 1022 & 4.89 & $0.002^{\#}$ \\
DSM Hyperactive-Impulsive & 1022 & 4.89 & $0.002^{\#}$ \\
DSM Total & 1022 & 5.83 & $0.001^{\#}$ \\
\hline
\end{tabular}

CBCL: Child Behavior Checklist - Revised; ADHD: attention-deficit/hyperactivity disorder; CPRS-R:

Conners' Parent Rating Scales - Revised; DSM: Diagnostic and Statistical Manual of Mental Disorders.

\# : omnibus comparison is significant after false discovery rate control for multiple comparisons [48]. 


\begin{tabular}{|c|c|c|c|c|}
\hline Test & Group 1 & Group 2 & Group 3 & Group 4 \\
\hline CBCL Internalising & $52.36 \pm 11.87$ & $57.29 \pm 10.19^{\#}$ & $55.80 \pm 11.59$ & $55.83 \pm 10.38$ \\
\hline ADHD & $58.92 \pm 8.50$ & $61.37 \pm 8.76^{\#, \eta_{1}+}$ & $58.29 \pm 8.06$ & $58.51 \pm 8.28$ \\
\hline Total & $54.32 \pm 12.49$ & $59.27 \pm 10.69^{\#}$ & $57.51 \pm 11.87$ & $57.20 \pm 11.06$ \\
\hline CPRS-R Cognitive & $51.64 \pm 10.74$ & $57.02 \pm 12.12^{\#, 9}$ & $55.72 \pm 12.35$ & $56.36 \pm 12.95$ \\
\hline Hyperactivity & $56.75 \pm 12.86$ & $60.85 \pm 12.93^{\uparrow,+}$ & $58.45 \pm 12.91$ & $57.44 \pm 12.14$ \\
\hline Psychosomatic & $50.79 \pm 11.35$ & $56.67 \pm 13.31^{\#}$ & $57.01 \pm 13.66$ & $57.63 \pm 13.04$ \\
\hline ADHD & $53.45 \pm 11.47$ & $58.84 \pm 12.37^{\#}$ & $56.88 \pm 12.58$ & $56.76 \pm 12.52$ \\
\hline DSM Inattentive & $51.70 \pm 11.03$ & $56.83 \pm 12.08^{\#}$ & $55.44 \pm 12.10$ & $54.64 \pm 12.30$ \\
\hline DSM Hyperactive-Impulsive & $56.61 \pm 12.62$ & $60.61 \pm 12.94^{\#}$ & $59.38 \pm 12.27$ & $58.79 \pm 12.58$ \\
\hline DSM Total & $54.10 \pm 11.86$ & $59.86 \pm 12.74^{\#}$ & $58.04 \pm 12.89$ & $57.12 \pm 12.77$ \\
\hline
\end{tabular}

Data are presented as mean士SD. CBCL: Child Behavior Checklist - Revised; ADHD: attention-deficit/ hyperactivity disorder; CPRS-R: Conners' Parent Rating Scales - Revised; DSM: Diagnostic and Statistical Manual of Mental Disorders. Reported p-values are adjusted using Bonferroni's correction. \#: Group 2 is significantly more impaired than Group 1 at $p<0.05$; ": Group 2 is significantly more impaired than Group 3 at $\mathrm{p}<0.05 ;{ }^{+}$: Group 2 is significantly more impaired than Group 4 at $p<0.05$.

\section{Discussion}

This study suggests that SDB during early childhood is a significant contributor to challenges with attention and behavioural regulation, as rated by parents. These findings follow on multiple previous studies, although with much more limited clinical samples [18-27]. Results indicate that behavioural challenges consistent with a potential diagnosis of $\mathrm{ADHD}$ are more common in children with SDB, including inattention, motor restlessness and hyperactivity, and associated behavioural dysregulation. Additionally, we found that mood difficulties were significantly noted, highlighting the potential impact of SDB on emotional regulation. However, of greater significance is the current finding that the impact of SDB on behaviour is greatest in children with the mildest degree of severity when compared with controls. This pattern was noted across both a broad-scale assessment, the CBCL, and one that is more directly associated with challenges seen in attentional disorders. Our results suggest that the potential alterations in sleep structure in habitually snoring children that are not readily identified by nocturnal PSG standard scoring approaches may exert effects on the underlying neural mechanisms that support aspects of both directed and sustained attention, as well as frontal-striatal circuits that are known to mediate emotion and behavioural regulation. Of note, the impact of intermittent hypoxia, as derived from the nadir arterial oxygen saturation measured by pulse oximetry $\left(\mathrm{SpO}_{2}\right)$ levels, was not as prominent, and suggests that the deleterious effects of the latter in the developing central nervous system may be located in different regions that may be more specifically involved in cognition and memory [47, 48]. Analyses identified that snoring and resultant sleep fragmentation also likely serve as the initial important stepwise increase in behavioural impact, such that increased SDB severity in conjunction with higher AHI and lower $\mathrm{SpO}_{2}$ fail to increase the magnitude of behavioural challenge. Although the observed behavioural trajectory across the SDB spectrum does not appear to overlap with the extent of neural substrate injury (i.e. as deduced from the observation that increased SDB severity leads to dose-dependent cognitive impact), it reminds us that a lower threshold regarding the expression of behavioural challenge in affected children may be present. Thus, more severe levels of SDB may in fact contribute to a reduced ability to regulate behaviour in conjunction with an impaired capacity to effectively muster the cognitive resources required to challenge and oppose efforts at external control; this conjectural explanation remains a focus for future research. Still, even in children with higher BMI z-scores, the prominent impact of snoring in the context of AHI $<1 \mathrm{hTST}^{-1}$ remained apparent when compared with higher SDB severity groups, thereby supporting the preferential role of sleep disruption in the processes underlying behavioural dysfunction among snoring children.

Our results were highly consistent across boys and girls. Additionally, initial screening analyses suggested that Group 2 had consistently greater behavioural pathology across all racial groups, but low numbers of children representing some races within defined sleep pathology groups resulted in inconsistencies in significance patterns in the present study. It is worth noting that additional considerations may exist regarding parental sensitivities toward behaviour problems across race and sex that should be considered in future research. Indeed, several studies have shown that parents differ in their ratings of their children's behavioural functioning across both socioeconomic status and race [49, 50]. Additionally, observations regarding behavioural presentation, as well as actual levels of behavioural output, differ in response to health and BMI status. Similarly, parents of boys tend towards stronger negative ratings in terms of attention and behavioural control [51]. 
An important clinically relevant take-home lesson emanates from these findings: children who snore may be at increased risk for developing and demonstrating attentional and behavioural regulatory concerns, independent of the severity of SDB. Both internalising and externalising challenges were noted to be of significant concern for children with SDB in this study. This highlights a need to address the risk for behavioural disruption in children who develop snoring; the latter is a likely precursor to ongoing challenges with both socialisation and regulation, and could, if unaddressed, adversely impact learning and social adaptation. However, our study does not answer the question concerning whether by addressing the underlying causes of snoring, the anticipation of improved functioning in behavioural control and learning will occur. Recent findings from the only randomised interventional trial for mild to moderate OSA would suggest that the more immediate impact of treatment is reflected in behaviour rather than cognition [24]. Similarly, children presenting clinically for evaluation of behavioural difficulties that may lead, on initial examination, to a consideration of ADHD should be clearly evaluated as to their sleep hygiene and snoring status. Based on current findings, the possibility exists that a significant number of children who are considered for stimulant treatment may actually benefit more directly from sleep assessment and intervention, with improved outcomes. The effectiveness of such interventions, and presence of residual OSA, may additionally depend on factors such as age and BMI [52, 53]. Consequently, the implementation of divergent clinical management approaches for snoring children with behavioural perturbations but otherwise normal sleep studies as compared with similarly snoring children in whom behavioural issues are absent will have to be scrutinised in future research.

In summary, the present study suggests that the presence of snoring, and inferentially of disrupted sleep, underlies the emergence of behavioural perturbations associated with inattention and impulsivity, and that further increases in the severity of SDB are not accompanied by concomitant increases in the severity of the behavioural patterns.

\section{Limitations}

One clear limitation to this study is the focus solely on parent reports regarding behavioural functioning, as opposed to assessing a broader environmental impact that would be afforded by additional teacher reports. Given the difficulties that are inherent in obtaining reports regarding behaviour from multiple individuals, a decision was made to rely principally on parents as informers. This decision obviously limits our understanding of the wider presentation of challenging behaviours that may exist for this sample. Furthermore, since both snoring status and behaviour problems were assessed through parent reports, it is possible that parents who suspected an association between snoring and behaviour problems could have introduced some recall bias into these comparisons. However, prior research has also demonstrated elevated behavioural problems in children with sleep disorders using teacher reports [54-57], which are less likely to be biased by knowledge of sleep status. Agreement between parent and teacher reports of behaviour may diverge at high levels of reported behavioural pathology, perhaps due to different environments under observation [57]. In addition, studies have reported high sensitivity and specificity for parent-reported snoring status in relation to PSG [58], and a general lack of parent knowledge of the association between SDB and behavioural problems [59].

An additional salient limitation of the current study is the low representation of nonsnoring African-American children, and the resulting inability to examine possible moderating effects of race on the relationship between SDB severity and behavioural outcomes. Previous research has been much more variable in regard to its sampling, with a focus on clinic-based referrals; the current study, while still showing some variability in the diversity of children included, is one of the largest population-based samples available to date and one that was collected separately from clinic referral. As such, while concerns regarding absolute representation remain, this study does in fact show the risk for impact of SDB within the broader paediatric population. Finally, despite the accumulating evidence of a strong association between SDB and behavioural outcomes, due caution should be exercised in inferring causal inferences from observational research.

\section{Future directions}

It will be important in future studies to assess the relationship between behavioural and cognitive variables, such as to potentially uncover a more ecologically based range of impact, and such aim is now a principal focus for future research. Additionally, we believe that integrating teacher reports may increase ecological understanding of behavioural impact and help guide stronger decision making regarding how to best, and when to best, intervene.

\section{Acknowledgements}

Authors contributions are as follows: D.L. Smith provided critical input to behaviour function interpretation and data analysis, and drafted the initial version of the manuscript; D. Gozal conceptualised the study, was responsible for sleep study interpretation, performed data analysis and edited the manuscript; S.J. Hunter performed individual behavioural data analysis and contributed to manuscript editing; M.F. Philby recruited subjects, scored and interpreted sleep studies, and participated in database maintenance and analysis; J. Kaylegian participated in sample collection as well as 
behavioural battery scoring; L. Kheirandish-Gozal conceptualised the study, recruited subjects, scored sleep studies, coordinated the database, analysed data and drafted critical portions of the manuscript; she is also the guarantor of the paper, taking responsibility for the integrity of the work as a whole, from inception to published article. All authors approved the final manuscript as submitted.

\section{References}

1 Kheirandish-Gozal L, Gozal D. Sleep Disordered Breathing in Children. 1st Edn. New York, Springer Science, 2012.

2 Gottlieb DJ, Vezina RM, Chase C, et al. Symptoms of sleep-disordered breathing in 5-year-old children are associated with sleepiness and problem behaviors. Pediatrics 2003; 112: 870-877.

3 Bonuck KA, Chervin RD, Cole TJ, et al. Prevalence and persistence of sleep disordered breathing symptoms in young children: a 6-year population-based cohort study. Sleep 2011; 34: 875-884.

4 Rosen CL, Larkin EK, Kirchner HL, et al. Prevalence and risk factors for sleep-disordered breathing in 8- to 11-year-old children: association with race and prematurity. J Pediatr 2003; 142: 383-389.

5 Montgomery-Downs HE, Gozal D. Sleep habits and risk factors for sleep-disordered breathing in infants and young toddlers in Louisville, Kentucky. Sleep Med 2006; 7: 211-219.

6 Bixler EO, Vgontzas AN, Lin HM, et al. Sleep disordered breathing in children in a general population sample: prevalence and risk factors. Sleep 2009; 32: 731-736.

7 Li AM, So HK, Au CT, et al. Epidemiology of obstructive sleep apnea syndrome in Chinese children: a two-phase community study. Thorax 2010; 65: 991-997.

8 Marcus CL, Brooks LJ, Draper KA, et al. Diagnosis and management of childhood obstructive sleep apnea syndrome. Pediatrics 2012; 130: e714-e755.

9 Weissbluth M, Davis AT, Poncher J, et al. Signs of airway obstruction during sleep and behavioral, developmental, and academic problems. J Dev Behav Pediatr 1983; 4: 119-121.

10 Ali NJ, Pitson D, Stradling JR. Sleep disordered breathing: effects of adenotonsillectomy on behaviour and psychological functioning. Eur J Pediatr 1996; 155: 56-62.

11 Urschitz MS, Eitner S, Guenther A, et al. Habitual snoring, intermittent hypoxia, and impaired behavior in primary school children. Pediatrics 2004; 114: 1041-1048.

12 Owens J, Opipari L, Nobile C, et al. Sleep and daytime behavior in children with obstructive sleep apnea and behavioral sleep disorders. Pediatrics 1998; 102: 1178-1182.

13 Guilleminault C, Winkle R, Korobkin R, et al. Children and nocturnal snoring: evaluation of the effects of sleep related respiratory resistive load and daytime functioning. Eur J Pediatr 1982; 139: 165-171.

14 Bonuck K, Freeman K, Chervin RD, et al. Sleep-disordered breathing in a population-based cohort: behavioral outcomes at 4 and 7 years. Pediatrics 2012; 129: e857-e865.

15 O'Brien LM, Lucas NH, Felt BT, et al. Aggressive behavior, bullying, snoring, and sleepiness in schoolchildren. Sleep Med 2011; 12: 652-658.

16 Giordani B, Hodges EK, Guire KE, et al. Neuropsychological and behavioral functioning in children with and without obstructive sleep apnea referred for tonsillectomy. J Int Neuropsychol Soc 2008; 14: 571-581.

17 Chervin $\mathrm{RD}$, Archbold KH, Dillon JE, et al. Inattention, hyperactivity, and symptoms of sleep-disordered breathing. Pediatrics 2002; 109: 449-456.

18 Kaplan BJ, McNicol J, Conte RA, et al. Sleep disturbance in preschool-aged hyperactive and nonhyperactive children. Pediatrics 1987; 80: 839-844.

19 Stein MA, Mendelsohn J, Obermeyer WH, et al. Sleep and behavior problems in school-aged children. Pediatrics 2001; 107: e60.

20 Chervin RD, Dillon JE, Bassetti C, et al. Symptoms of sleep disorders, inattention, and hyperactivity in children. Sleep 1997; 20: 1185-1192.

21 Chervin RD, Archbold KH. Hyperactivity and polysomnographic findings in children evaluated for sleep-disordered breathing. Sleep 2001; 24: 313-320.

22 O'Brien LM, Mervis CB, Holbrook CR, et al. Neurobehavioral implications of habitual snoring in children. Pediatrics 2004; 114: 44-49.

23 O'Brien LM, Mervis CB, Holbrook CR, et al. Neurobehavioral correlates of sleep-disordered breathing in children. J Sleep Res 2004; 13: 165-172.

24 Marcus CL, Moore RH, Rosen CL, et al. A randomized trial of adenotonsillectomy for childhood sleep apnea. New Engl J Med 2013; 368: 2366-2376.

25 Gottlieb DJ, Vezina RM, Chase C, et al. Symptoms of sleep-disordered breathing in 5-year-old children are associated with sleepiness and problem behaviors. Pediatrics 2003; 112: 870-877.

26 Montgomery-Downs HE, Jones VF, Molfese VJ, et al. Snoring in preschoolers: associations with sleepiness, ethnicity, and learning. Clin Pediatr 2003; 42: 719-726.

27 Melendres MC, Lutz JM, Rubin ED, et al. Daytime sleepiness and hyperactivity in children with suspected sleep-disordered breathing. Pediatrics 2004; 114: 768-775

28 Corkum $\mathrm{P}$, Tannock $\mathrm{R}$, Moldofsky $\mathrm{H}$, et al. Actigraphy and parental ratings of sleep in children with attention-deficit/hyperactivity disorder (ADHD). Sleep 2001; 24: 303-312.

29 Corkum P, Tannock R, Moldofsky H. Sleep disturbances in children with attention-deficit/hyperactivity disorder. J Am Acad Child Adolesc Psychiatry 1998; 37: 637-646.

30 O'Brien LM, Holbrook CR, Mervis CB, et al. Sleep and neurobehavioral characteristics of 5- to 7-year-old children with parentally reported symptoms of attention-deficit/hyperactivity disorder. Pediatrics 2003; 111: 554-563.

31 Beebe DW, Gozal D. Obstructive sleep apnea and the prefrontal cortex: towards a comprehensive model linking nocturnal upper airway obstruction to daytime cognitive and behavioral deficits. J Sleep Res 2002; 11: 1-16.

32 Barnes ME, Gozal D, Molfese DL. Attention in children with obstructive sleep apnoea: an event-related potentials study. Sleep Med 2012; 13: 368-377.

33 Barnes ME, Huss EA, Garrod $\mathrm{KN}$, et al. Impairments in attention in occasionally snoring children: an event-related potential study. Dev Neuropsychol 2009; 34: 629-649.

34 Beebe DW. Cognitive, behavioral, and functional consequences of inadequate sleep in children and adolescents. Pediatr Clin North Am 2011; 58: 649-665. 
35 Spruyt K, Gozal D. Sleep disturbances in children with attention-deficit/hyperactivity disorder. Expert Rev Neurother 2011; 11: 565-577.

36 Fallone G, Owens JA, Deane J. Sleepiness in children and adolescents: clinical implications. Sleep Med Rev 2002; 6: 287-306.

37 Sans Capdevila O, Crabtree VM, Kheirandish-Gozal L, et al. Increased morning brain natriuretic peptide levels in children with nocturnal enuresis and sleep disordered breathing: a community-based study. Pediatrics 2008; 121: e1208-e1214.

38 Spruyt K, Gozal D. Screening of pediatric sleep disordered breathing: a proposed unbiased discriminative set of questions using clinical severity scales. Chest 2012; 142: 1508-1515.

39 Achenbach TM. Manual for the Child Behavior Checklist/4-18 and 1991 Profile. Burlington, University Associates in Psychiatry, 1991.

40 Nakamura BJ, Ebesutani C, Bernstein A, et al. A psychometric analysis of the Child Behavior Checklist DSM-oriented scales. J Psychopathol Behav Assess 2009; 31: 178-189.

41 Conners CK. Conners' Rating Scales - Revised. North Tonawanda, Multi-Health Systems, 1997.

42 Conners CK, Sitarenios G, Parker JD, et al. The revised Conners' Parent Rating Scale (CPRS-R): factor structure, reliability, and criterion validity. J Abnorm Child Psychol 1998; 26: 257-268.

43 Chang LY, Wang MY, Tsai PS. Diagnostic accuracy of rating scales for attention-deficit/hyperactivity disorder: a meta-analysis. Pediatrics 2016; 137: 1-13.

44 Montgomery-Downs HE, O’Brien LM, Gulliver TE, et al. Polysomnographic characteristics in normal preschool and early school-aged children. Pediatrics 2006; 117: 741-753.

45 Berry RB, Budhiraja R, Gottlieb DJ, et al. Rules for scoring respiratory events in sleep: update of the 2007 AASM Manual for the Scoring of Sleep and Associated Events. Deliberations of the Sleep Apnea Definitions Task Force of the American Academy of Sleep Medicine. J Clin Sleep Med 2012; 8: 597-619.

46 Kaditis AG, Alonso Alvarez ML, Boudewyns A, et al. Obstructive sleep disordered breathing in 2- to 18-year-old children: diagnosis and management. Eur Respir J 2016; 47: 69-94.

47 Row BW, Kheirandish L, Neville JJ, et al. Impaired spatial learning and hyperactivity in developing rats exposed to intermittent hypoxia. Pediatr Res 2002; 52: 449-453.

48 O'Brien LM, Tauman R, Gozal D. Sleep pressure correlates of cognitive and behavioral morbidity in snoring children. Sleep 2004; 27: 279-282.

49 Piotrowska PJ, Stride CB, Groft SE, et al. Socioeconomic status and antisocial behavior among children and adolescents: a systematic review and meta-analysis. Clin Psychol Rev 2015; 35: 47-55.

50 Petersen IT, Bates JE, Dodge KA, et al. Describing and predicting developmental profiles of externalizing problems from childhood to adulthood. Dev Psychopathol 2015; 27: 791-818.

51 Graves SL, Blake J, Kim ES. Differences in parent and teacher ratings of preschool problem behavior in a national sample: the significance of gender and SES. J Early Intervention 2012; 34: 151-165.

52 Alonso-Álvarez ML, Terán-Santos J, Navazo-Egüia AI, et al. Treatment outcomes of obstructive sleep apnoea in obese community-dwelling children: the NANOS study. Eur Respir J 2015; 46: 717-727.

53 Kaditis A, Lévy P. Focus on prevention and treatment of obstructive sleep disordered breathing in childhood. Eur Respir J 2015; 46: 615-618.

54 Ali NJ, Pitson DJ, Stradling JR. Snoring, sleep disturbance, and behaviour in 4-5 year olds. Arch Dis Child 1993; 68: $360-366$.

55 Arman AR, Ersu R, Save D, et al. Symptoms of inattention and hyperactivity in children with habitual snoring: evidence from a community-based study in Istanbul. Child Care Health Dev 2005; 31: 707-717.

56 Beebe DW, Wells CT, Jeffries J, et al. Neuropsychological effects of pediatric obstructive sleep apnea. J Int Neuropsychol Soc 2004; 10: 962-975.

57 Kohler MJ, Kennedy JD, Martin AJ, et al. Parent versus teacher report of daytime behavior in snoring children. Sleep Breath 2013; 17: 637-645.

58 Montgomery-Downs HE, O Brien LM, Holbrook CR, et al. Snoring and sleep-disordered breathing in young children: subjective and objective correlates. Sleep 2004; 27: 87-94.

59 Strocker AM, Shapiro NL. Parental understanding and attitudes of pediatric obstructive sleep apnea and adenotonsillectomy. Int J Pediatr Otorhinolaryngol 2007; 71: 1709-1715. 